\title{
Secondary haemophagocytic lymphohistiocytosis in hospitalised COVID-19 patients as indicated by a modified HScore is infrequent and high scores do not associate with increased mortality
}

\author{
Authors: Michael R Ardern-Jones, ${ }^{\mathrm{A}}$ Matt Stammers, ${ }^{\mathrm{B}}$ Hang TT Phan, ${ }^{\mathrm{C}}$ Florina Borca, ${ }^{\mathrm{D}}$ Anastasia Koutalopoulou, ${ }^{\mathrm{E}}$ \\ Ying Teo, ${ }^{\mathrm{E}}$ James Batchelor, ${ }^{\mathrm{F}}$ Trevor Smith ${ }^{G}$ and Andrew S Duncombe ${ }^{H}$
}

\section{A significant proportion of COVID-19 patients show} evidence of hyperinflammation (HI), of which secondary haemophagocytic lymphohistiocytosis (sHLH) is the most severe manifestation and diagnosed with HScore. Using a COVID-relevant modification of the HScore (\%HScore), we set out to determine the prevalence of sHLH in 567 COVID-19 inpatient cases.

The overall incidence of individuals with an $80 \%$ probability of sHLH in our COVID-19 cohort was $1.59 \%$ on admission and only rose to $4.05 \%$ if calculated at any time during admission. This small cohort as defined by \%HScore showed no excess mortality compared with the whole cohort. Overall, \%HScores were lower in older patients $(p<0.0001)$ and did not reliably predict outcome at any cut-off value (AUROC 0.533, $p=0.211$, odds ratio 0.99 ).

Authors: Aassociate professor, University of Southampton, Southampton UK, and consultant dermatologist, University Hospital Southampton NHS Foundation Trust, Southampton, UK; ${ }^{B}$ consultant gastroenterologist, University Hospital Southampton NHS Foundation Trust, Southampton, UK and visiting research fellow, University of Southampton, Southampton, UK; ' data science lead, University of Southampton, Southampton, UK, and data science fellow, NIHR Southampton Biomedical Research Centre, University Hospital Southampton NHS Foundation Trust, Southampton, UK; Dsenior information analyst for research \& development, University of Southampton, Southampton, UK, and data science fellow, NIHR Southampton Biomedical Research Centre, University Hospital Southampton NHS Foundation Trust, Southampton, UK; ${ }^{E}$ clinical fellow, University Hospitals Southampton NHS Foundation Trust, Southampton, UK; Fdirector, Clinical Informatics Research Unit, University of Southampton, Southampton, UK, and senior investigator, NIHR Southampton Biomedical Research Centre, University Hospital Southampton NHS Foundation Trust, Southampton, UK; ${ }^{6}$ consultant gastroenterologist, University Hospital Southampton NHS Foundation Trust, Southampton, UK; ${ }^{H}$ consultant haematologist, University Hospital Southampton NHS Foundation Trust, Southampton, UK
Our study demonstrates that a modified version (\%HScore) of the conventional sHLH scoring system (HScore) does not enable risk stratification in people hospitalised with COVID. We propose further work is needed to develop novel approaches to predict $\mathrm{HI}$ and improve trial stratification for $\mathrm{HI}$ directed therapy in people with COVID-19.

KEYWORDS: Hyperinflammation, SARS-CoV-2, HScore, secondary haemophagocytic lymphohistiocytosis, COVID-19

DOI: $10.7861 /$ clinmed.2021-0053

Introduction

Mortality from SARS-CoV-2 infection causing COVID-19 in hospitalised patients in the UK has been reported to be $25.7 \%$. 'The principal cause of death due to COVID-19 is respiratory failure due to acute respiratory distress syndrome. ${ }^{2}$ Early reports have suggested that a subgroup of individuals suffer a hyperinflammatory state with high mortality which is associated with high levels of IL- 6 and C-reactive protein (CRP). ${ }^{3}$ Hyperinflammation ( $\mathrm{HI}$ ) has been previously described secondary to acute infection and termed cytokine release syndrome / cytokine storm (CRS/CS), macrophage activation syndrome (MAS), macrophage-cytokine self-amplifying loop (MCSAL) and secondary haemophagocytic lymphohistiocytosis ( $\mathrm{SHLH}$ ). HI in COVID-19 has drawn attention because of overlapping features with these classical syndromes, notably high fever, striking acute phase response and coagulopathy. Yet despite the descriptions of these overlapping conditions, characterised by a rapid increase in systemic inflammation, there remains no consensus as to the precise definition of what constitutes HI. ${ }^{4}$ However, early reports to date suggest that in COVID-19 the inflammatory response, as indicated by ferritin and CRP levels, is lower overall than in classical $\mathrm{HI}$ syndromes such as SHLH. ${ }^{5} \mathrm{HI}$ in COVID-19 may either be a different inflammatory cascade to that induced by $\mathrm{sHLH}$, or could possibly reflect differences in the spectrum of $\mathrm{HI}$ severity. Therefore, it is of interest to determine the prevalence of sHLH in people with COVID-19. In addition, we have applied modified sHLH criteria (\%Hscore) to a cohort of people hospitalised 
with COVID-19 to determine whether such analysis illuminates debate around the HI disease spectrum, as has been previously suggested. ${ }^{6}$

While it is accepted that viral infections are the commonest cause of sHLH, ${ }^{7}$ symptoms of $\mathrm{HI}$ resemble those of general sepsis; therefore $\mathrm{HI}$ has generally been under-recognised at an early stage, leading to high mortality. ${ }^{8}$ It is likely that strategies to identify $\mathrm{HI}$ and targeted intervention will offer the most effective approach to the management of HI in COVID-19. Indeed, as well as anti-IL- $6,{ }^{9}$ other cytokines released in $\mathrm{HI}$ for which existing biologic therapies are available could also represent potential targets for intervention; these potential therapies include inhibitors of TNF- $\alpha$ (infliximab), IL-1 (anakinra), and JAK (eg ruxolitinib). Randomised controlled trial data have shown that the anti-inflammatory agent dexamethasone can reduce mortality in severe COVID-19 in an unselected cohort ${ }^{1}$ and targeted anti-inflammatory anti-IL- 6 therapy in an unselected intensive care COVID-19 population showed reduced mortality in the intervention vs control arms (22.2-28\% vs $35.8 \%$, respectively). ${ }^{10}$ While impressive, these results suggest that targeted antiinflammatory interventions given early to individuals with HI may show even greater benefit in mortality, and this approach may be the key to reducing the morbidity of COVID-19 by preventing escalation to high dependency and intensive care. To facilitate diagnosis of $\mathrm{sHLH}$, the most extreme form of $\mathrm{HI}$, the 'HScore' 111,12 has been developed because of evidence that early recognition and intervention is beneficial (Table 1).12 While the HScore has some limitations, ${ }^{13}$ including that it was not validated on a critical care population, and that despite its use $\mathrm{SHLH}$ is still underrecognised because of the complexity of the syndrome, some authors have recommended using the HScore in COVID-19. ${ }^{14} \mathrm{~A}$ recent report using this approach has provided evidence that the prevalence of $\mathrm{SHLH}$ is low $(7.5 \%)$ in intensive care patients with COVID-19 $(n=40)$. We therefore set out to examine the HScores in people hospitalised with COVID-19, and to explore the prevalence of sHLH as assessed by a COVID-modified $\mathrm{H}$ score across the whole hospitalised COVID-19 cohort.

\section{Patients and methods}

Following national ethical approval (Identification of Novel Factors Leading to Activated Macrophage Expansion in COVID19 and related conditions to guide targeted intervention, Inflame COVID-19 Study, NRES 286016) which included retrospective collection of virus-induced SHLH controls, we recruited all cases of COVID-19 infection that tested positive for SARS-CoV-2 viral RNA in our laboratory and were admitted to University Hospitals Southampton NHS Foundation Trust between 7 March 2020 and 9 June 2020 ( $n=626$ ). Additionally, we recruited a retrospective cohort of sHLH (viral infection associated) from the same institution based on confirmed diagnosis recorded as ICD-10 D76.2 $(n=16)$.

Structured and semi-structured data were accrued from the trust integration engine using SQL Developer 4.2 queries and then cleaned/transformed using python 3.7 and associated libraries: numpy and pandas. Analysis was performed using matplotlib, seaborn and scipy. Statistical analysis was undertaken using GraphPad, Prism (8.4.3)

The classical HScore (Table 1) includes three clinical parameters (immunosuppression, pyrexia, organomegaly), five blood tests
Table 1. HScore and \%HScore algorithm

\begin{tabular}{|c|c|c|}
\hline Parameter & $\begin{array}{l}\text { HScore points } \\
\text { (criteria) }\end{array}$ & $\begin{array}{l}\text { \%HScore points } \\
\text { (criteria) (Minimum } \\
\text { variables } 3 \text { ) }\end{array}$ \\
\hline Temperature $\left({ }^{\circ} \mathrm{C}\right)$ & $\begin{array}{l}0(<38.4) \\
33(38.4-39.4) \\
\text { or } 49(>39.4)\end{array}$ & $\begin{array}{l}0(<38.4) \\
33(38.4-39.4) \\
\text { or } 49(>39.4)\end{array}$ \\
\hline No of cytopenias* & $\begin{array}{l}0 \text { (one lineage), } \\
24 \text { (two lineages), } \\
\text { or } 34 \text { (three lineages) }\end{array}$ & $\begin{array}{l}0 \text { (one lineage), } \\
24 \text { (two lineages), or } \\
34 \text { (three lineages) }\end{array}$ \\
\hline Ferritin (mg/L) & $\begin{array}{l}0(<2,000), \\
35(2,000-6,000), \\
\text { or } 50(>6,000)\end{array}$ & $\begin{array}{l}0(<2,000), \\
35(2,000-6,000) \text {, } \\
\text { or } 50(>6,000)\end{array}$ \\
\hline Triglyceride (mmol/L) & $\begin{array}{l}0(<1.5), 44(1.5-4) \\
\text { or } 64(>4)\end{array}$ & $\begin{array}{l}0(<1.5), 44(1.5-4), \\
\text { or } 64(>4)\end{array}$ \\
\hline Fibrinogen ( $\mathrm{g} / \mathrm{L})$ & $\begin{array}{l}0(>2.5) \text { or } \\
30(\leq 2.5)\end{array}$ & $\begin{array}{l}0(>2.5) \text { or } \\
30(\leq 2.5)\end{array}$ \\
\hline AST/ALT (IU/L) & $0(<30)$ or $19(\geq 30)$ & $0(<30)$ or $19(\geq 30)$ \\
\hline Haemophagocytosis $^{+}$ & 0 (no) or 35 (yes) & - \\
\hline Immunosuppression & 0 (no) or 18 (yes) & - \\
\hline $\begin{array}{l}\text { Hepatomegaly / } \\
\text { splenomegaly }\end{array}$ & $\begin{array}{l}0 \text { (none), } 23 \text { (either), } \\
\text { or } 38 \text { (both) }\end{array}$ & - \\
\hline Score & $\begin{array}{l}\text { Sum of points above } \\
\text { (maximum 337) }\end{array}$ & $\begin{array}{l}\text { Sum of points } \\
\text { above / maximum } \\
\text { score (maximum } \\
100 \% \text { ) }\end{array}$ \\
\hline
\end{tabular}

*Haemoglobin $\leq 92 \mathrm{~g} / \mathrm{L}$ and/or WBC $\leq 5 \times 10^{9} / \mathrm{L}$ and/or platelets $\leq 110 \times 10^{9} / \mathrm{L}$. 'Features on bone marrow aspirate. ALT = alanine aminotransferase; $\mathrm{AST}=$ aspartate transaminase; IU = international units.

(triglyceride, ferritin, transaminase, fibrinogen, cytopenia), and bone marrow aspirate features. Each of these is weighted by variable and a score based on the value/result is summated to provide an overall score ranging from $0-337$. This value is then utilised to calculate a probability of a diagnosis of $\mathrm{HLH}$; for example, an $\mathrm{HScore}$ of $\leq 90$ equates to a $<1 \%$ probability of $\mathrm{SHLH}$, while there is a $>99 \%$ probability with an $\mathrm{HScore}$ of $\geq 250$. We calculated the HScore based on parameters available retrospectively. As expected from the infective precautions taken on COVID-19 patients, or from the lack of clinical indication for the investigation, few data were available on palpable hepatosplenomegaly or bone marrow aspirate histology, and on analysis we found the electronic data on immunosuppression status to be unreliable. Therefore, we excluded these three parameters. To account for these missing values we created a modified HScore calculated from the percentage points from the available parameters expressed as a percentage ( $\%$ HScore, Table 1 ).

The primary outcome utilised in this study was binary: discharge from hospital or death in hospital. Admission date was an unreliable marker of disease onset as some of our cohort contracted COVID-19 after prolonged periods in hospital and therefore the time of initial infection was unclear. Clinical teams arranged testing as symptoms presented and therefore, to facilitate comparison between cases, investigation parameters 


\begin{tabular}{ll}
\hline Table 2. COVID cohort characteristics and comorbidities \\
\hline Age (median years, IQR) & $71(54-82)$ \\
Sex: male (\%), female (\%) & $58.20,41.80$ \\
BAME (\%) & 11.46 \\
BMI (mean, IQR) & $25.73(22.49-30.22)$ \\
Comorbidities (\%) & \\
Cardiac disease & 71.78 \\
Renal disease & 59.96 \\
Respiratory disease & 41.09 \\
Gastrointestinal disease & 38.10 \\
Diabetes & 26.28 \\
Neurological disease & 51.15 \\
Cancer history & 28.04 \\
\hline BAME = Black, Asian and minority ethnic; BMI $=$ body mass index; IQR $=$ \\
interquartile range.
\end{tabular}

were normalised to the date ofSARS-CoV-2 viral RNA laboratory confirmation and outcome data tabulated from day -1 to day 21 .

\section{Results}

Considering the influence of age on mortality, we examined our dataset for the number of recorded HScore parameters (day -1 to $4)$, as distributed by age $(n=621)$ (supplementary material S1a). This showed that the individuals for whom few data points were available were more likely to be older $(p=0.0025)$. To address this source of potential bias, we removed individuals with fewer than three data points from further analysis. Subsequent analysis of the distribution of data points in the reduced cohort $(n=567)$ confirmed no association between the number of data parameters and age $(p=0.094)$, confirming that the analysis was valid across all age groups. The characteristics of the 567 eligible cases $(41.8 \%$ female) showed a high prevalence of comorbidities in line with the high overall average age (median 71 years, interquartile range [IQR] 54-82; Table 2).

As expected, because of missing data, the classical HScores in our cohort were low (maximum 147, equivalent to $43.6 \%$ of the maximal possible HScore). However, \%HScore measured in the first 5 days of illness (day -1 to 4 after laboratory virus confirmation) was a very strong predictor of the $\% \mathrm{HScore}$ during the whole admission ( $r=0.8499, p<0.0001$, Fig 1a), and good correlation was observed between \%HScore and classical HScore $\left(r^{2}=0.88\right.$; see supplementary material S1b). Interestingly, examined in isolation, none of the parameters in the \%HScore measured at day -1 to 4 differentiated those who would survive or die except for white cell count, where those who survived versus died showed a lower mean value ( 6.63 vs $8.27 \times 10^{9} / \mathrm{L}, p=0.000071$; false discovery rate $<1 \%$; see supplementary material S1c).

Compared to the SHLH cohort, COVID-19 showed a significantly lower \% HScore (median $73.47 \%$ vs $18.13 \%$ respectively, $\mathrm{p}<0.0001$, Fig 1b). An HScore which predicts an $80 \%$ probability of sHLH is reported to be $191 / 337^{11}$ which is equivalent to a \% HScore of $56.7 \%$. If \% HScore was calculated from 'worst' values at any time day -1 to 21 , the proportion of COVID-19 cases meeting the sHLH threshold was only marginally higher at $4.05 \%$ (23 of 567). At the early time point (virus day -1 to 4 ), these criteria were met by only $1.59 \%$ ( 9 of 567) COVID-19 cases. Surprisingly, for those
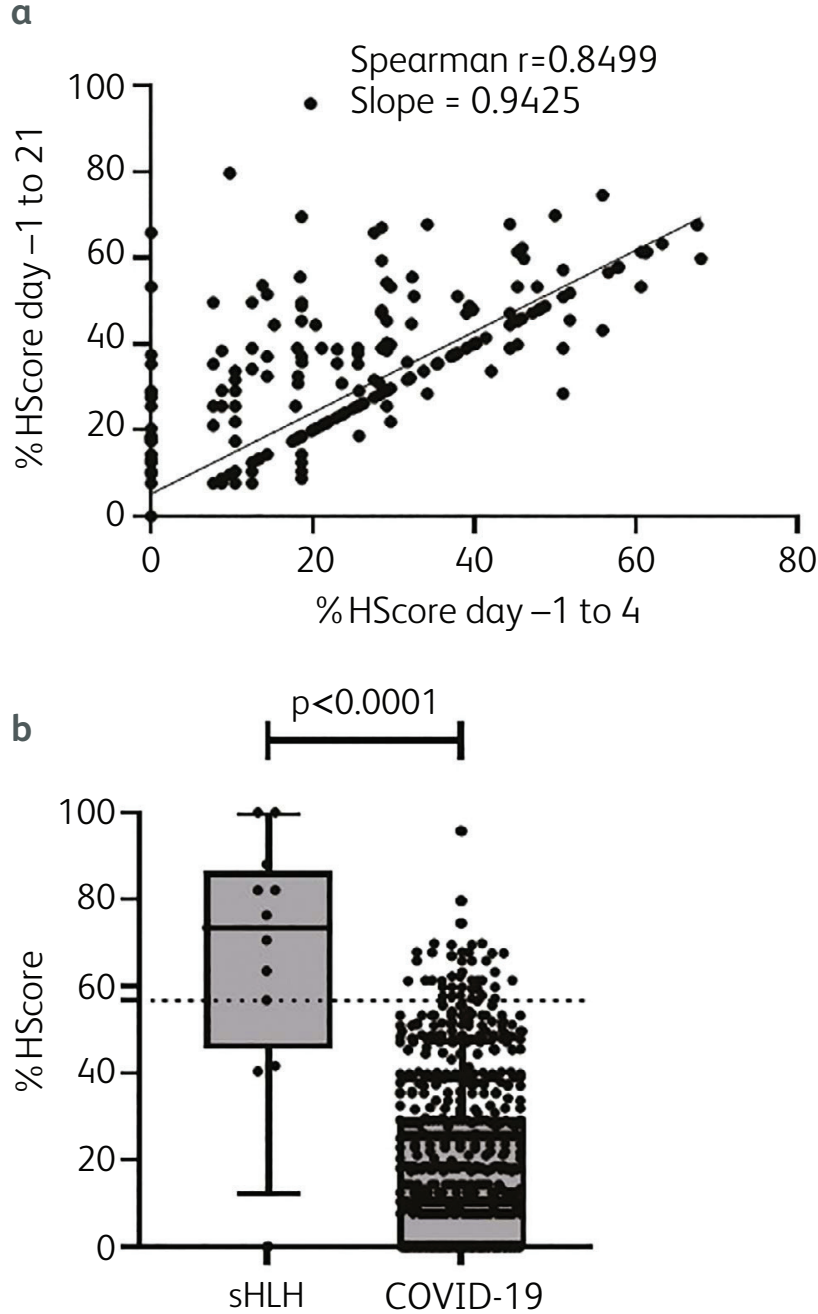

Fig 1. sHLH shows a higher \%HScore than COVID-19, but \%HScore in COVID-19 shows no correlation with mortality. a) \%HScore as measured from data points recorded at virus diagnosis timepoints day -1 to 4 , versus day -1 to 21 . Spearman's correlation coefficient ( $r$ ) presented. $\mathrm{n}=567$. b) Plot of $\% \mathrm{HScores}$ from a retrospective cohort of secondary haemophagocytic lymphohistiocytosis (sHLH) versus COVID-19. Dotted line at $56.7 \%(80 \%$ probability of HLH). Error bars represent $10-90 \%$ confidence. Mann Whitney test presented.

individuals with a \% HScore above the $\mathrm{SH} \mathrm{LH}$ threshold, there was no increase in mortality as compared to the whole cohort mortality of $30.43 \%$ vs $30.69 \%$ respectively ( $p>0.05$ ).

In order to determine the role of \%HScore for early identification of $\mathrm{HI}$ across the whole cohort, we restricted analysis to scoring from day -1 to 4 , and then correlated this early measure with mortality at any time point. As seen in many studies in COVID-19, overall mortality was strongly predicted by patient age $(p<0.0001$; median age survivors 64 years, IQR 49-76; mean age of those who died 81 years, IQR 73-87; see supplementary material S2a). At a threshold of 75 years of age, the increased risk of mortality was significant (odds ratio [OR] 7.295, 4.89-10.8, p<0.0001). However, age conferred a strong negative correlation on $\%$ HScore (Spearman $r=-0.305,-0.38$ to $-0.226, p<0.0001$; see supplementary material S2b), across the cohort. Strikingly, 
the median \%HScore was significantly lower $(p<0.0001)$ in the older age group: $>75$ years median \%HScore $7.724(0.0-18.16)$ vs $\leq 75$ years median \%HScore 18.31 (7.72-28.57) (Fig 2a). Receiver operator characteristics (ROC) over the whole cohort suggest that

a
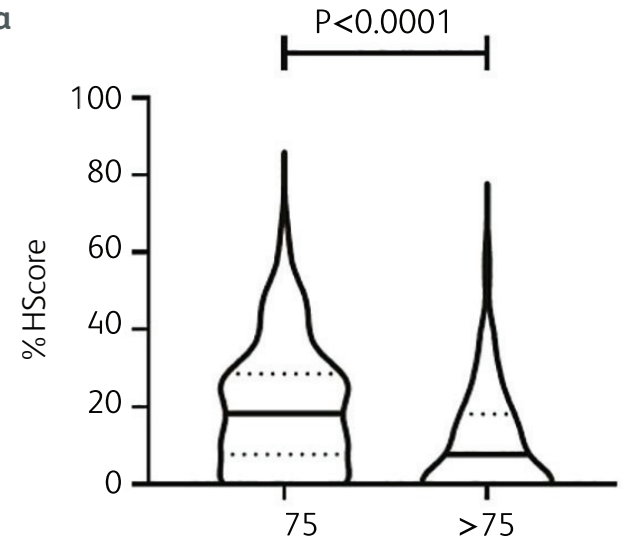

\begin{tabular}{c|c|c} 
& $\leq 75$ & $>75$ \\
\hline Median & 18.31 & 7.724
\end{tabular}

b

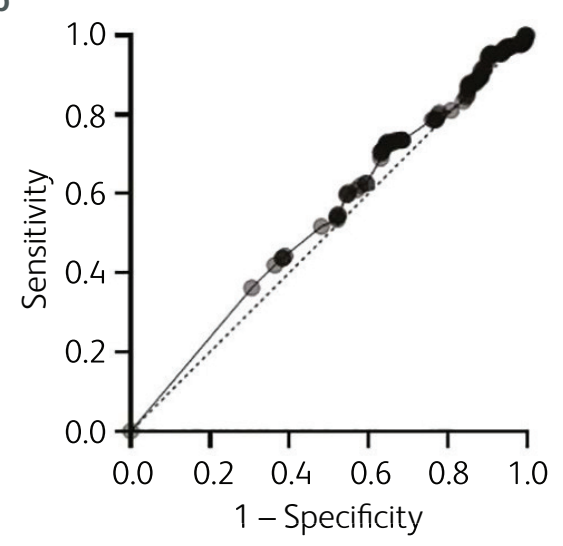

C

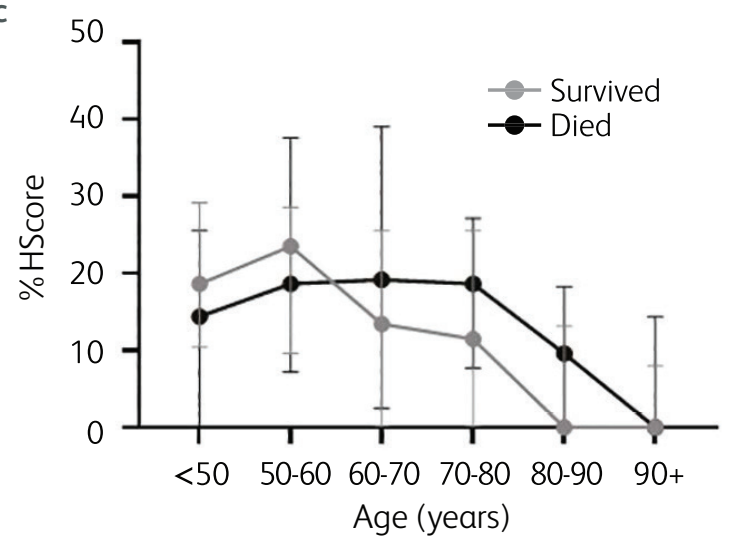

Fig 2. Age, \%HScore and risk of mortality in COVID-19. a) Violin plot of $\% H S c o r e$ in those $\leq 75$ versus $>75$ years $(n=567)$. Horizontal bars represent median value, interquartile range dotted. Mann Whitney statistic presented. b) Receiver operator characteristics of prediction of mortality by $\% \mathrm{HScore}$. c) \%HScore in cases who died (black dots) versus survived (grey dots) by age stratification. Error bars represent $25-75 \%$ confidence interval. at any threshold, \%HScore is not useful as a predictor of mortality in COVID-19 (AUROC 0.533, $p=0.211$; OR 0.99, 0.98-1.00) (Fig 2b). However, because of the very strong association between age and mortality, it is important to stratify for age to examine the effect of \%HScore on mortality. Stratification showed that the negative correlation between age and \% HScore was highly significant in both those who survived $(r=-0.307,-0.441$ to $-0.164, p<0.0001)$ and those who died $(r=-0.309,-0.441$ to $-0.164, p<0.0001)$ and that there was no difference in \%HScore between those who died and survived ( $p=0.3125$ ) (Fig 2c).

\section{Discussion}

We report here the largest dataset assessing $\mathrm{SHLH}$ incidence by $\%$ HScore in COVID-19 to date $(n=567)$, which exceeds the 312 sHLH cases in the original series identifying the $\mathrm{HScore}^{11}$ and the 40 cases where HScore was applied to intensive care patients. ${ }^{13}$ During COVID-19, inevitably some parameters in the HScore were not obtainable, and our study demonstrates that use of the $\mathrm{HScore}$ during the pandemic is challenging. However, to address missing data, we utilised a mathematical programmed approach to facilitate rigorous data collection from centralised hospital electronic records and utilised cross-checking and cross-validation to optimise data cleaning, thus avoiding collection errors, while minimising missing data. Furthermore, to identify the subgroup with sHLH in COVID-19, we undertook a stringent approach to the analysis and did not impute any missing values and instead designed a COVID-modified HScore, \% HScore.

In this report, we demonstrate that $\mathrm{SHLH}$, as measured by the $\% \mathrm{HScore}$, is rare in hospitalised cases of COVID-19, similar to the reports of low incidence in intensive care settings. ${ }^{13,14}$ Indeed, we estimate that SHLH arises in $1.59 \%$ of hospitalised COVID-19 cases early in the course of the illness, and only rising to $4.7 \%$ over the whole admission. Surprisingly, mortality in the \%HScoresHLH cohort of COVID-19 cases meeting $80 \%$ probability showed no excess mortality as compared to the whole cohort $(30.43 \%$ vs $30.69 \%$ ). We emphasise some caution when translating this finding to cases diagnosed by the traditional HScore because of the natural limitations of undertaking this work in a pandemic meant that the full quota of HScore parameters (including, for example, the presence of haematophagocytosis on bone marrow aspirate findings) was impossible to attain on any COVID patient. Therefore, the cases with high \%HScores here may not necessarily have achieved a similar HScore. In addition, this analysis did not stratify for therapy and it remains possible that medical interventions may have modified the mortality of the cases with higher scores. We stress that COVID-19 patients demonstrating high likelihood of sHLH should still be treated with standard treatment protocols for $\mathrm{SHLH}^{15}$

It is notable that the index cohort of SHLH cases used to define the HScore had a median age of 51 years (IQR 36-64), ${ }^{11}$ as compared to our COVID-19 patients whose median age was 71 years (IQR 54-82). In addition, we identified that younger patients have significantly higher \%HScores $(p<0.0001)$ and additionally show that when stratified for age, there was no difference in $\% \mathrm{HScore}$. Why \%HScore (and HScore parameters) decline with age in the context of COVID-19 is not clear but may predominantly reflect immunosenescence. In part this may be explained by responses to COVID-19 generally acting in an opposite direction to HLH. For example, while pancytopaenia would produce a higher 
$\% \mathrm{HScore}$, it seems that responses to the virus in older individuals are more likely to show increases in circulating white blood cells and platelets, which would clearly drive the \%HScore down. Therefore, the association between reduced \% HScore and age, as well as the relatively low mortality of SHLH in COVID-19, suggests that waning immunity with age may actually be protective against sHLH-type responses in COVID-19.

Although it is possible that high \%HScores in COVID-19 do reflect dysregulated immunity, the absolute difference between those who die and survive is small, suggesting that the individual with a high \%HScore may lie at or close to a tipping point between harm and benefit from innate inflammation. Therefore, it remains unclear what the effect of broadly applied anti-inflammatory therapies will have on older individuals in particular and a careful balance needs to be struck when designing clinical trials of antiinflammatory therapies to determine where an individual lies on the risk spectrum of an excessive inflammatory response versus an impaired anti-viral response. Improved endotyping of COVID-19 cases by classification of validated biochemical and molecular phenotypes to identify the subgroup who will benefit from anti-HI strategies is critical and these should be used to stratify COVID-19 patients in the next phase of clinical trials; early reports look promising. ${ }^{16}$ This emphasises that interventional approaches need to be guided by deep understanding of the inflammatory processes underway at an individual patient level. Some efforts have also been made to develop markers of $\mathrm{HI}$, but the index cohorts remain small. ${ }^{17}$

In summary, we present data which show that when applying a modified HScore (\%HScore), $\mathrm{SHLH}$ is uncommon in hospitalised cases of COVID-19 and in cases where scores are higher, this does not predict outcome. Why \%HScore (and most HScore parameters) decline with age in the context of COVID-19 is not clear but may predominantly reflect immunosenescence in this mainly elderly cohort of patients. We suggest that waning immunity with age may actually be protective against $\mathrm{sHLH}$ type responses in COVID-19 patients. However, several studies have shown the benefit of anti-HI therapy in COVID-19 patient cohorts (dexamethasone in oxygen-dependent patients and tociluzimab in ITU patients). We show here that the conventional scoring system for sHLH will not identify the group who are most likely to benefit from such therapy. Indeed, while the work here shows that in COVID-19, sHLH is uncommon, this work does not undermine the utility of the HScore as a diagnostic tool for $\mathrm{SHLH}$ in COVID-19 and we encourage readers to actively manage sHLH in accordance with international guidance..$^{15}$ Our study demonstrates the importance for novel algorithms to predict $\mathrm{HI}$ in COVID-19 as well as randomised controlled trials targeted at this patient group.

\section{Supplementary material}

Additional supplementary material may be found in the online version of this article at www.rcpjournals.org/clinmedicine: S1 - Analysis of available HScore parameters versus age. S2 - The effect of age on overall survival and \%HScore in COVID-19.

\section{References}

1 The RECOVERY Collaborative Group. Dexamethasone in hospitalized patients with Covid-19 - preliminary report. N Engl ] Med 2020;384:693-704.

2 Huang C, Wang Y, Li X et al. Clinical features of patients infected with 2019 novel coronavirus in Wuhan, China. Lancet 2020;395:497-506.

3 Ruan Q, Yang K, Wang W, Jiang L, Song J. Clinical predictors of mortality due to COVID-19 based on an analysis of data of 150 patients from Wuhan, China. Intensive Care Med 2020;46:846-8.

4 McGonagle D, Ramanan AV, Bridgewood C. Immune cartography of macrophage activation syndrome in the COVID-19 era. Nat Rev Rheumatol 2021:17:145-57.

5 Chen N, Zhou M, Dong X et al. Epidemiological and clinical characteristics of 99 cases of 2019 novel coronavirus pneumonia in Wuhan, China: a descriptive study. Lancet 2020;395:507-13.

6 Castillo L, Carcillo J. Secondary hemophagocytic lymphohistiocytosis and severe sepsis/ systemic inflammatory response syndrome/multiorgan dysfunction syndrome/macrophage activation syndrome share common intermediate phenotypes on a spectrum of inflammation. Pediatr Crit Care Med 2009;10:387-92.

7 Carter SJ, Tattersall RS, Ramanan AV. Macrophage activation syndrome in adults: recent advances in pathophysiology, diagnosis and treatment. Rheumatology 2019;58:5-17.

8 Okabe T, Shah G, Mendoza V et al. What intensivists need to know about hemophagocytic syndrome: an underrecognized cause of death in adult intensive care units. J Intensive Care Med 2012;27:58-64.

9 Xu X, Han M, Li T et al. Effective treatment of severe COVID-19 patients with tocilizumab. Proc Natl Acad Sci USA 2020;117:10970-5.

10 Gordon AC, Mouncey PR, Al-Beidh F et al. Interleukin-6 receptor antagonists in critically ill patients with Covid-19. N Engl J Med 2021;384:1491-1502.

11 Fardet L, Galicier L, Lambotte $\mathrm{O}$ et al. Development and validation of the HScore, a score for the diagnosis of reactive hemophagocytic syndrome. Arthritis Rheumatol 2014:66:2613-20.

12 Debaugnies F, Mahadeb B, Ferster A et al. Performances of the $\mathrm{H}$-Score for diagnosis of hemophagocytic lymphohistiocytosis in adult and pediatric patients. Am J Clin Pathol 2016;145:862-70.

13 Wood $\mathrm{H}$, Jones J, Hui K et al. Secondary HLH is uncommon in severe COVID-19. Br J Haematol 2020;90:e283-e285.

14 Mehta P, McAuley DF, Brown M et al. COVID-19: consider cytokine storm syndromes and immunosuppression. Lancet 2020;395:1033.

15 La Rosee P, Horne A, Hines $\mathrm{M}$ et al. Recommendations for the management of hemophagocytic lymphohistiocytosis in adults. Blood 2019;133:2465-77.

16 Manson J], Crooks C, Naja M et al. COVID-19-associated hyperinflammation and escalation of patient care: a retrospective longitudinal cohort study. Lancet Rheumatol 2020;2:e594-e602.

17 Webb BJ, Peltan ID, Jensen P et al. Clinical criteria for COVID-19associated hyperinflammatory syndrome: a cohort study. Lancet Rheumatol 2020;2:e754-e63.

Address for correspondence: Dr Michael R Ardern-Jones, Clinical Experimental Sciences, University of Southampton Faculty of Medicine, Mailpoint 825, Level F, South Block, Sir Henry Wellcome Laboratories, Southampton General Hospital, Southampton SO16 6YD, UK.

Email:m.aj@soton.ac.uk

Twitter: @MikeAJderm 